\title{
Welche strategischen Kompetenzen brauchen Patienten im Gesundheitswesen der Zukunft?
}

\author{
Das Trägernetzwerk von Kompetenzentwicklung in Humanmedizin (KHM-cap) will \\ das Konzept Health Literacy verstehen und Chancen und Risiken der Entwicklung \\ von Gesundheitskompetenz sehen. Dazu fand am 6./7. Mai das 3. KHM-Zukunfts- \\ forum [1]* statt. Entstanden sind Kompetenzanforderungsprofile für vier Patienten- \\ gruppen im Jahre 2030, die mit diesem Thesenpapier erstmals öffentlich zur Dis- \\ kussion gestellt werden.
}

Christoph A. Pfister ${ }^{a}$
Therese Stutz Steiger $^{b}$

a Dr. med., Master of Medical Education (UniBE), Facharzt Anästhesiologie, Kompetenz entwicklung in Humanmedizin (KHM-cap)

b Dr. med., Master of Public Health

Interessenbindungen: Christoph A. Pfister ist im Bereich Kompetenzentwicklung selbständig erwerbend tätig und lizenzierter $\mathrm{KODE}^{\circledR}$-/ KODE ${ }^{\circledR} \mathrm{X}$-Trainer. Die Tätigkeit vorliegende Arbeit wurden finanziell nicht unterstützt. Therese Stutz Steiger ist im Bereich Public Health selbständig erwerbend tätig und Co-Präsidentin der Schweizerischen Vereinigung Osteogenesis Imperfecta (SVOI). Seit 2011 ist sie Vorstandsmitglied der Allianz Seltener Krankheiten Schweiz ProRaris. und insbesondere die

«Gesundheitskompetenz basiert auf allgemeiner Kompetenz und beinhaltet Fähigkeiten, Fertigkeiten, Wissen und Motivation, um im Alltag relevante Gesundheitsinformationen zu finden, zu verstehen, zu beurteilen und anzuwenden, um in den Bereichen Gesundheitsförderung, Krankheitsprävention und Krankheitsbewältigung Entscheidungen zu treffen und zu handeln, mit dem Ziel, gute Lebensqualität während des gesamten Lebenslaufs zu erhalten und zu verbessern.» Diese umfassende Definition wurde aus einer Analyse von 17 Annäherungen an den Begriff hergeleitet [2].

Health Literacy bzw. Gesundheitskompetenz kann der Bevölkerung die Entwicklung einer zukunftsbewussten Gesundheitsorientierung ermöglichen [3]. Die Fähigkeit zur Informationsverarbeitung zwecks Entscheidungsfähigkeit in der Familie, im Alltag, als Konsument(in) auf dem (Gesundheits-/Krankheits-)Markt und im Arbeitsleben hat einen hohen Stellenwert [4].

Als wichtige Beteiligte gelten in der Schweiz drei Gruppen [5]:

- Einzelpersonen, Familien, Gruppen und Gemeinschaften

- Fachpersonen und Institutionen (u.a. Gesundheitssystem, Wirtschaftssystem, Bildungssystem)

- Behörden und Gesundheitspolitik

Korrespondenz:

Dr. med. Christoph A. Pfister Kompetenzentwicklung in Humanmedizin (KHM-cap) Hauptstrasse 66 CH-3286 Muntelier Tel. 0266706028 www.khm-cap.ch
Das Thesenpapier entstand unter aktiver Mitwirkung folgender Personen (alphabetische Reihenfolge):

Franziska Baumann, Bern

Prof. Volker Heyse, Regensburg

Patrizia Kündig, Chur

Lydia Rufer-Drews, Meiringen

Pia Sangiorgio, Münchenbuchsee

PD Dr. med. et MME (UniBE) Matthias Widmer, Bern

\section{Thèses de la société KHM-cap à} I'occasion du Zukunftsforum 2014 consacré à la compétence du patient

Health Literacy (ou compétence informationnelle en santé) est le terme anglais internationalement utilisé pour permettre entre autres à l'être humain d'accroître sa compétence décisionnelle dans le domaine de la santé. Aujourd'hui, l'acquisition de compétences est le facteur-clé du système économique. Grâce à l'outil $K O D E^{\circledR} X$, il a été possible de déterminer pour les patients du futur les compétences-clés qui leurs permettront d'utiliser efficacement le système de santé en 2030 . Celles-ci se fondent sur cinq thèses: le patient doit être au centre et est mieux à même de puiser dans ses propres compétences lorsqu'il est face à un professionnel de la santé qui le traite d'égal à égal. Autrement dit, il s'agit d'accueillir le patient avec une nouvelle attitude professionnelle. Tous les individus doivent développer la solidarité et la sensibilité nécessaires pour participer au développement de processus politiques essentiels pour la santé. Parmi les 12 compétences-clés, l'autogestion est la plus importante. De plus, quatre compétences-clés différenciées ont été mises en évidence pour les personnes atteintes de maladies aiguës, les malades chroniques, les patients souffrant de maladies rares et des patients potentiels. Les offres de formation et les processus de changement dans le domaine de la santé peuvent s'appuyer sur ces facteurs prometteurs. 
Mit fünf Thesen soll in den nächsten Monaten die Diskussion mit den Akteuren in der Schweiz und im nahen deutschsprachigen Ausland gesucht werden. Es geht dabei um die Fragen

1. Welche Rolle spielt der/die Patient(in) in Zukunft?

2. Wohin führt ein Machtgefälle zwischen Patient(inn)en und Health Professionals?

3. Was wird sich für Health Professionals in $\mathrm{Zu}$ kunft ändern?

4. Was muss sich für alle Menschen in der Schweiz ändern?

5. Welche Schlüsselkompetenzen sind für welche Patientengruppen prioritär?

\section{These: Der/die Patient(in) gehört ins Zentrum} des Gesundheitswesens.

Etwas provokativ führte diesen Satz eine in doppeltem Sinne Betroffene weiter: ... und dort ist er im Weg! [6] Um dies zu ändern sind beide Seiten gefordert:

Dazu gehört, dass Patient(inn)en nicht übergangen, sondern als vollwertig wahrgenommen werden, ja sogar den Fokus bilden für alle im Gesundheitswesen Tätigen. Es bedeutet aber auch, dass Patient(inn)en dazu beitragen, dass das Gesundheitssystem effektiv funktioniert [7].

Dies stützt auch das 2. von 4 Zielen der «Gesundheit2020»-Strategie des Bundesrates [8]:

- Chancengleichheit und Selbstverantwortung stärken, insbesondere Versicherte und Patient(inn)en stärken (Punkt 2.3)

In der Folge fördert der Bund bei der «Stiftung Patientensicherheit Schweiz» den verstärkten Einbezug der Patient(inn)en [9].

Die Akademien der Wissenschaften Schweiz haben ebenfalls 2012 eine Roadmap für ein nachhaltiges Gesundheitssystem mit sieben Zielen formuliert. Das siebte heisst:

- Sowohl Public Health als auch die Eigenverantwortung des Bürgers sind gestärkt (Gesundheitskompetenz/Health Literacy) [10].

Aber nicht nur der Staat, auch die Wirtschaft will mehr Patienten-/Kundenorientierung: Mit dem wachsenden Gesundheitsmarkt und der zunehmenden Bedeutung von Prävention und Gesundheitsförderung wandelt sich seine Rolle hin zum Konsumenten und Bürger. Das Gesundheitssystem ist jedoch komplex und verlangt vom Bürger eine aktivere Rolle [11].

In Annäherung an die Patientenperspektive wird klar, dass dieser Patient / diese Patientin der Zukunft über umfassende Kenntnisse und Fähigkeiten verfügen muss. Selbstmanagementprogramme für chronische Patient(inn)en verbreiten sich zunehmend [12]. Die Frage ist, ob dies genügt, um beispielsweise mit dem grossen Marketing-Druck der Industrie umgehen zu können? Die Unterscheidung von wissenschaftlich fundiertem Fachwissen und MarketingInformationen ist selbst für Health Professionals eine Herausforderung.
2. These: Patient(inn)en können die eigenen Kompetenzen besser ausschöpfen, wenn sie Health Professionals auf Augenhöhe begegnen. Ausgeliefert- oder Abhängig-Sein schränkt die Handlungsfähigkeit ein. Möglichst viele Menschen sollen die verschiedenen Stadien der individuellen Lebensund Patientenrolle mit ihren vielen herausfordernden Übergangsphasen mit einem Bewusstsein der aktiven Beteiligung (als Akteur) erleben. Gesundheitsfachleute können allerdings in diesem Prozess der Autonomieförderung eine entscheidende Rolle spielen [13]. Die Interaktion zwischen Patient(in) und Health Professionals (HP) soll sich im Rahmen des Möglichen vermehrt auf gleicher Augenhöhe abspielen, z. B. im Rahmen von Symposien oder Patientenforen.

\section{These: Dem Patienten / der Patientin der Zukunft soll mit einer neuen professionellen Haltung begegnet werden.}

Gegenseitiger Respekt vor den Kompetenzen des Gegenübers fördert die Motivation für ein sinnvolles und effizientes Selbst- und Organisationsmanagement. Die Beteiligung von Patient(inn)en an den wichtigen Zukunftsfragen rund um das Gesundheitswesen, insbesondere unter Berücksichtigung von neuen Technologien und des Informationsmanagements, wird diese zielführend beeinflussen. Die Zusammenarbeit soll geprägt sein von der Qualität des Informationsaustausches, der Würdigung von Laienkenntnissen, der Nutzung der vorhandenen Ressourcen und der Fähigkeit der HP, komplexe Sachverhalte einfach auszudrücken [14].

\section{These: Das Individuum soll Solidarität und} Sensibilität für die Mitgestaltung von gesundheitsrelevanten politischen Prozessen entwickeln. Es wäre wünschenswert, dass Bürger(innen) (und Patient[inn]en) vermehrt in die Erarbeitung von Gesundheitsstrategien und in klinische Projekte einbezogen würden. In England ist das beispielsweise seit 2005 ein Programmpunkt, der auf höchster Ebene verfolgt wird [15]. Im schweizerischen System könnten innovative Wege gesucht werden.

Es sei jedoch auch an vulnerable Gruppen erinnert, die über wesentlich geringere Gesundheitskompetenzen verfügen und häufiger ärztliche Hilfe in Anspruch nehmen müssen, häufiger die Notfallpforte aufsuchen, häufiger hospitalisiert werden und häufiger Medikamenten- und Behandlungsfehler erleben. Sie brauchen spezielle Informationen und angepasste Unterstützung.

\section{These: Patientenkompetenzen beschreiben die Selbstorganisationsdisposition von Menschen für die erfolgreiche Nutzung des Gesundheits- systems.}

Patient(inn)en werden 1. als (noch) gesunde Bürger(innen) (potentielle Patient[innen]), die mit Prä- 
mien und Steuern das Gesundheitssystem finanzieren, 2. als notfallmässig erkrankte, 3. als chronisch kranke oder 4. als Patient(inn)en mit seltenen Erkrankungen in unterschiedlichen Stadien/Lebensphasen oder Rollen angetroffen. Sie sind mit einem zunehmend komplexeren Gesundheitssystem und einer explodierenden Menge an Informationen konfrontiert.

Die Anforderungen an die Menschen in diesen Rollen, aber auch an das System sind sehr unterschiedlich. Unter Nutzung des Kompetenz-Explorers $\left(\mathrm{KODE}^{\circledR} \mathrm{X}\right)$ [16], der auch für die Humanmedizin [17] verwendet wird, wurden in folgender Reihenfolge zwölf Schlüsselkompetenzen als Grundstock für alle Gruppen festgelegt:

1. Selbstmanagement

2. Eigenverantwortung

3. Entscheidungsfähigkeit

4. Offenheit für Veränderungen

5. Optimismus

6. Beurteilungsvermögen

7. Folgebewusstsein

8. Initiative

\section{Abbildung 1}

KompetenzAtlas mit Markierung der 12 Basiskompetenzen (graues Dreieck) und jeweils 4 spezifische Kompetenzen für 4 Gruppen: akut Erkrankte (rot), chronisch Kranke (blau), Patient(inn)en mit seltener Erkrankung (gelb) und potentielle Patient(inn)en (grün).
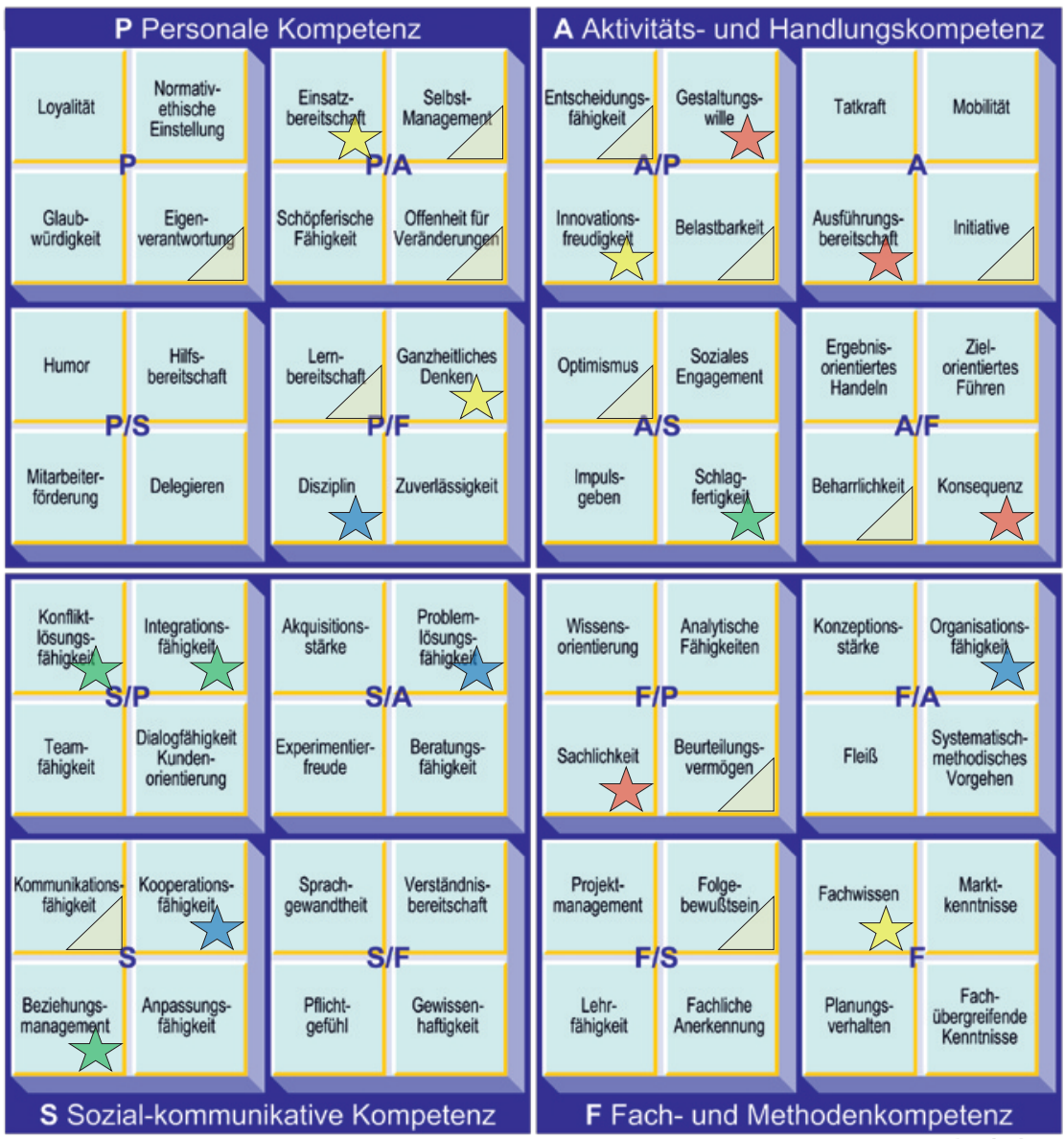

9. Beharrlichkeit

10. Belastbarkeit

11. Lernbereitschaft

12. Kommunikationsfähigkeit

Das Selbstmanagement ist also nur eine von zwölf strategischen Kompetenzen, wenn auch die wichtigste. Für jede Patientengruppe sind vier weitere, spezifische Schlüsselkompetenzen wesentlich (Abb. 1):

Gesunde(r) Bürger(in) (grün)

- Beziehungsmanagement

- Integrationsfähigkeit

- Konfliktlösungsfähigkeit

- Schlagfertigkeit

Notfallmässig erkrankte(r) Patient(in) (rot)

- Ausführungsbereitschaft

- Gestaltungswille

- Konsequenz

- Sachlichkeit

Chronisch kranke(r) Patient(in) (blau)

- Disziplin

- Kooperationsfähigkeit

- Organisationsfähigkeit

- Problemlösungsfähigkeit

Patient(in) mit seltener Erkrankung (gelb)

- Einsatzbereitschaft

- Fachwissen

- Ganzheitliches Denken

- Innovationsfreudigkeit

Diese Auswahl von strategischen Kompetenzen wurde durch eine breit zusammengesetzte Gruppe von unterschiedlichen HP, Public Health-Expert(inn)en, Patient(inn)en und Angehörigen ermittelt. Für die Präzisierung der Kompetenzbeschriebe dienen die Vorlagen in $\mathrm{KODE}^{\circledR}$ und die ausführlichen Ergänzungen für die Humanmedizin [18].

\section{Nächste Schritte}

Parallel zur Publikation des Thesenpapiers sind Gespräche geplant mit Organisationen des Gesundheitswesens: Vertreter(innen) der Health Professionals, Spitex, Patientenorganisationen, Bildungsstrukturen u.a. Die Erkenntnisse sollen die laufenden Entwicklungen zielgerichtet unterstützen: Bildungsangebote und Change-Prozesse im Gesundheitswesen können sich an diesen erfolgversprechenden Faktoren orientieren. Zentral dafür ist eine breite Diskussion zu den fünf Thesen, zu der die Leser und Leserinnen der SÄZ herzlich eingeladen sind.

* Die Literatur findet sich online unter www.saez.ch $\rightarrow$ Aktuelle Ausgabe oder $\rightarrow$ Archiv $\rightarrow 2014 \rightarrow 41$ 


\section{Literatur}

1 Kickbusch I, Pelikan JM, Apfel F, Tsouros AD. Health Literacy - The Solid Facts. In: Europe ROf, ed. Copenhagen: WHO; 2013:86.

2 Sørensen K, Van den Broucke S, Fullam J, et al. Health literacy and public health: a systematic review and integration of definitions and models. BMC public health. 2012;12:80.

3 Stutz Steiger T. Gesundheitskompetenz: Stärkung des Gesundheitssystems durch Stärkung der Menschen (http://ch.linkedin.com/pub/stutz-steiger-therese/15/ b16/788) Referat in ppt, gehalten am 1.3.2013 im Möschberg (2. KHM-Zukunftsforum).

4 Stutz Steiger T. Wo steht Health Literacy/Gesundheitskompetenz heute? Referat in ppt, gehalten am 6.5.2014 im Möschberg (3. KHM-Zukunftsforum).

5 Lehmann P, Stutz Steiger T. Förderung der Gesundheitskompetenz - Erhebung der Aktivitäten der Kantone. Bern: Allianz Gesundheitskompetenz; 2011.

6 Sax A. «Der Patient steht im Zentrum, und dort steht er im Weg!» Interview mit Therese Stutz Steiger. Schweiz Ärztezeitung. 2013;94:710-11.

7 Pelikan JM. Gesundheitskompetenz - Die HLS-EU Studie und ihre Folgen für die Gesundheitspolitik in Österreich. In: Symposium «Zukunft Gesundheitskompetenz Schweiz». 5.12.2013; Bern: Allianz für Gesundheitskompetenz; 2013.

8 Gesundheit2020 - Die gesundheitspolitischen Prioritäten des Bundesrates. In: Bundesrat (Hrsg.) Bern: Bundesamt für Gesundheit; 2013:25.

9 Erläuternder Bericht zum Bundesgesetz über das Zentrum für Qualität in der obligatorischen Krankenpflegeversicherung (Stärkung von Qualität und Wirtschaftlichkeit). In: EDI EDdI (Hrsg.). Bern: Schweizerische Eidgenossenschaft; 2014:75.
10 Ein nachhaltiges Gesundheitssystem für die Schweiz - Roadmap der Akademien der Wissenschaften Schweiz. Roadmap. Bern: Akademien der Wissenschaften Schweiz; 2012 Dez 04.

11 Wild S, Roediger A. Von der veränderten Rolle des Patienten - Gesundheitskompetenz als Schlüsselfaktor. In: Oggier W, Walter A, Reichlin S, Egli M (Hrsg.) Handbuch Gesundheitswesen Schweiz. Sursee: Trend Care AG; 2008.

12 Haslbeck J. Selbstmanagementförderung - Empowerment zu gesundheitsbewusstem Leben mit chronischer Krankheit am Beispiel von EVIVO. Care Management. 2012;5:23-6.

13 Stutz Steiger T. Gesundheitskompetenz - ein Thema auch für die Weiterbildung. Education Permanente. 2011:4-6.

14 Lorig K, Haslbeck J, Gabriel E, Natau C. Gesund und aktiv mit chronischer Krankheit leben. 2nd ed: Careum Verlag; 2013.

15 www.involve.org.uk/blog/2014/02/03/story-of-the-uknational-action-plan-2013-15/

16 Systematisches Kompetenzmanagement $\left(\mathrm{SKoM}^{\circledast}\right)$ von Volker Heyse (www.act-skom.de).

17 Heyse V, Schircks A. Kompetenzprofile in der Humanmedizin. Konzepte und Instrumente für die Ausrichtung von Aus- und Weiterbildung und Rekrutierung auf Schlüsselkompetenzen. Münster: Waxmann Verlag; 2012.

18 Heyse V, Pfister CA, Schircks A. KompetenzAtlas Humanmedizin (Schweiz). In: Heyse V, Schircks A (Hrsg.). Kompetenzprofile in der Humanmedizin Konzepte und Instrumente für die Ausrichtung von Aus- und Weiterbildung auf Schlüsselkompetenzen Münster: Waxmann; 2012:154-216. 